Article

\title{
Effective Null Raychaudhuri Equation
}

\author{
Alessandro Pesci ${ }^{(D)}$ \\ INFN Bologna, Via Irnerio 46, I-40126 Bologna, Italy; pesci@bo.infn.it
}

Received: 24 September 2018; Accepted: 19 October 2018; Published: 23 October 2018

\begin{abstract}
The effects on Raychaudhuri's equation of an intrinsically-discrete or particle nature of spacetime are investigated. This is done through the consideration of null congruences emerging from, or converging to, a generic point of spacetime, i.e., in geometric circumstances somehow prototypical of singularity issues. We do this from an effective point of view, that is through a (continuous) description of spacetime modified to embody the existence of an intrinsic discreteness on the small scale, this adding to previous results for non-null congruences. Various expressions for the effective rate of change of expansion are derived. They in particular provide finite values for the limiting effective expansion and its rate of variation when approaching the focal point. Further, this results in a non-vanishing of the limiting cross-sectional area itself of the congruence.
\end{abstract}

Keywords: quantum gravity; Raychaudhuri equation; effective metric

Recently, an effective metric, or qmetric, bitensor $q_{a b}$ has been introduced [1-3], capable of implementing the existence of an intrinsic discreteness or particle nature of spacetime at the microscopic scale, while keeping the benefits of a continuous description for calculus [4]. $q_{a b}$ acts like a metric in that it provides a (modified) squared distance between two generic spacelike or timelike separated events $P$ and $p$ (considered as the base and field point, respectively), which approaches the squared distance as of an ordinary $g_{a b}$ metric when $P$ and $p$ are far away. Contrary to a metric however, the squared distance approaches $\epsilon L^{2}$ (with $\epsilon=1(-1)$ for spacelike (timelike) separation) in the coincidence limit $p \rightarrow P$, with $L$ being an invariant length characterizing the qmetric.

In [5], an extension of this qmetric approach to include the case of null separated events has been considered, and an expression of $q_{a b}$ for them has been provided. This case could be directly relevant for the study of horizons. In the case of null geodesics near a focal point, this might be exploited for example to study event horizons at their birth (described, e.g., in [6] (in particular, Figure 57), [7], Figure 34.7, and [8], Box 12.1). When these geodesics are meant as histories of ultrarelativistic or massless particles, we are led to singularity formation issues. In view of this, the aim of this note is to investigate how the null Raychaudhuri equation gets modified by intrinsic discreteness of spacetime, as captured by the qmetric, near a focal point.

A wide range of results has been obtained in the past concerning the study of quantum effects on the Raychaudhuri equation. We would mention in particular the results obtained in Loop Quantum Gravity/Cosmology (LQG/LQC) $[9,10]$, which provide a detailed account, under isotropic conditions, of the resolution of Schwarzschild's singularity, as well as of the avoidance of the Big Bang singularity formation. In a different vein, the studies originated in [11] are somehow prototypical of attempts to include quantum effects in the Raychaudhuri equation with no reference to any specific quantum theory of gravity. These latter studies are successful, as well, in showing that quantum effects protect against singularity formation. The present attempt has also no reference to any definite quantum theory of gravity. The difference with [11] is in the way quantum effects are introduced: there, through consideration of quantum trajectories as in Bohm's pilot wave formulation of quantum mechanics; here, upon assuming the existence of a finite lower-limit invariant length $L$ between space- or time-separated events. The present study elaborates on previous results concerning the effects $L$ induces on the rate of change of expansion for timelike/spacelike congruences [12]. 
In [1-3], the qmetric is introduced as something that leads to replacing the quadratic distance $\sigma^{2}(p, P)$ between spacelike/timelike separated events by an effective distance $\left[\sigma^{2}\right]_{q}=S_{L}\left(\sigma^{2}\right)$ dependent on the characterizing scale $L$. This effective distance is subject to the requirements $S_{L} \rightarrow \epsilon L^{2}$ when $\sigma^{2} \rightarrow 0$ and $S_{L} \sim \sigma^{2}$ when $\sigma^{2} / L^{2}$ is large, as well as to an additional request in the form of the effective kernel $[G]_{q}$ of the d'Alembertian, namely that $[G]_{q}\left(\sigma^{2}\right)=G\left(S_{L}\right)$ in all maximally-symmetric spacetimes. This fixes the expression of $q_{a b}(p, P)$ to the form:

$$
q_{a b}=A g_{a b}+\epsilon\left(\frac{1}{\alpha}-A\right) t_{a} t_{b}
$$

where $t^{a}$ is the normalized tangent vector $\left(g_{a b} t^{a} t^{b}=\epsilon ; t_{a}=g_{a b} t^{b}\right)$ at $p$ to the geodesics connecting $P$ and $p, g_{a b}$ is considered at $p$ and $\alpha$ and $A$ are functions of $\sigma^{2}$, given by:

$$
\begin{gathered}
\alpha=\frac{S_{L}}{\sigma^{2} S_{L}^{\prime 2}}, \\
A=\frac{S_{L}}{\sigma^{2}}\left(\frac{\Delta}{\Delta_{S}}\right)^{\frac{2}{D-1}} .
\end{gathered}
$$

Here, the prime symbol indicates differentiation with respect to $\sigma^{2}$, and $\Delta$ is a van Vleck determinant ([13-16]; see [17-19]):

$$
\Delta(p, P)=-\frac{1}{\sqrt{g(p) g(P)}} \operatorname{det}\left[-\nabla_{a}^{(p)} \nabla_{b}^{(P)} \frac{1}{2} \sigma^{2}(p, P)\right]
$$

$\left(g=\operatorname{det} g_{a b}\right)$ and $\Delta_{S}(p, P)=\Delta(\tilde{p}, P)$ with $\tilde{p}$ being that point on the geodesic through $P$ and $p$ (on the same side of $p)$ with $\sigma^{2}(\tilde{p}, P)=S_{L}(p, P)$.

The extension of this approach to include the null case [5] is done shifting the focus of attention from quadratic distance, which is identically vanishing in this case, to affine parameterization. Exploiting the fact that an affine parameter $\lambda$, assigned with a null geodesics $\gamma$, is a distance as measured along $\gamma$ by suitable canonical observers parallelly-transported along it, the qmetric is introduced as something that leads to replacing $\lambda(p, P)$ (having $\lambda(P, P)=0$ ) with an effective parameterization $[\lambda]_{q}=\tilde{\lambda}(\lambda)$, which depends on the characterizing scale $L$ (we omit the explicit indication of this dependence). The effective parameterization has the requirements $\tilde{\lambda} \rightarrow L$ when $\lambda \rightarrow 0$ and $\tilde{\lambda} \sim \lambda$ when $\lambda / L$ is large, as well as the same additional request in the form of the effective kernel $[G]_{q}$ of the d'Alembertian as above, specialized to points on null geodesics. This last request consists of what is derived for points null separated from $P$ from requiring $[G]_{q}\left(\sigma^{2}\right)=G\left(S_{L}\right)$ in all maximally-symmetric spacetimes. This gives, for $q_{a b}(p, P)$ with $P$ and $p$ null separated, the expression:

$$
q_{a b}=A_{\gamma} g_{a b}-\left(\frac{1}{\alpha_{\gamma}}-A_{\gamma}\right) l_{(a} m_{b)}
$$

with $l^{a}=\frac{d x^{a}}{d \lambda}$ and $m^{a}$ null with $g_{a b} m^{a} l^{b}=-2$ considered at $p$ (as well as $g_{a b}$ is), $l_{a}=g_{a b} l^{b}, m_{a}=g_{a b} m^{b}$, and $\alpha_{\gamma}$ and $A_{\gamma}$ are functions of $\lambda$ given by:

$$
\begin{gathered}
\alpha_{\gamma}=\frac{1}{(d \tilde{\lambda} / d \lambda)^{2}}, \\
A_{\gamma}=\frac{\tilde{\lambda}^{2}}{\lambda^{2}}\left(\frac{\Delta}{\Delta_{S}}\right)^{\frac{2}{D-2}}\left(\frac{d \tilde{\lambda}}{d \lambda}\right)^{-\frac{2}{D-2}} .
\end{gathered}
$$


Here, $\Delta_{S}(p, P)=\Delta(\tilde{p}, P)$, where $\tilde{p}$ is that point on $\gamma$ (on the same side of $p$ ) that has $\lambda(\tilde{p}, P)=\tilde{\lambda}$ with $\left(\partial^{a} \sigma^{2}\right)_{\mid \tilde{p}}=\partial^{a} S_{L}=2 \tilde{\lambda} l_{\mid \tilde{p}}^{a}$.

The functions $\alpha_{\gamma}$ and $A_{\gamma}$ are defined for points on the null geodesic from $P$ and then only on the submanifold $\Gamma$ consisting of the null congruence of all null geodesics emerging from $P$ (considered as the base point). Crucial in the derivation of these expressions is considering the d'Alembertian at points of $\Gamma$ in a form that has no derivations of the vectors tangent to the congruence taken along directions outside $\Gamma$ [5]. This has been accomplished through the following expression for the d'Alembertian (meant as applied to a generic function $f\left(\sigma^{2}\right)$ in a maximally-symmetric spacetime):

$$
\square f=\nabla_{a} \nabla^{a} f=\left(4+2 \lambda \nabla_{i} l^{i}\right) \frac{d f}{d \sigma^{2}}
$$

$\left(i=1, \ldots, D-1\right.$ are indices of components on $\Gamma$ ), i.e., in terms of a quantity, $\nabla_{i} l^{i}=\theta$, the expansion of $\Gamma$, in which all variations are in $\Gamma$. Expressions of $\left[\nabla_{i} l^{i}\right]_{q}$ have then been readily obtained as:

$$
\begin{aligned}
{\left[\nabla_{i} l^{i}\right]_{q} } & =\nabla_{i}\left(\frac{d \lambda}{d \tilde{\lambda}} l^{i}\right)+\frac{1}{2} \frac{d \lambda}{d \tilde{\lambda}} q^{b c} l^{a} \nabla_{a} q_{b c} \\
& =\frac{d \lambda}{d \tilde{\lambda}} \nabla_{i} l^{i}-\frac{d \lambda}{d \tilde{\lambda}} \frac{d}{d \lambda} \ln \frac{d \lambda}{d \tilde{\lambda}}+\frac{1}{2}(D-2) \frac{d \lambda}{d \tilde{\lambda}} \frac{d}{d \lambda} \ln A_{\gamma},
\end{aligned}
$$

where $q^{a b}$ is the inverse of $q_{a b}$. These expressions provide the expansion $[\theta]_{q}$ of the null congruence $\Gamma$ according to the qmetric. The aim of this brief report is to discuss what the associated effective null Raychaudhuri equation is and to explore both this and the effective expansion $[\theta]_{q}$ at coincidence limit $p \rightarrow P$. The results we obtain refer to a null congruence emerging from generic $P$, but can equivalently be read as referring to a null congruence converging to $P$ upon substitution $\lambda \rightarrow-\lambda, \tilde{\lambda} \rightarrow-\tilde{\lambda}$ and $L \rightarrow-L$.

We begin by noting that, if we use the expressions for $\alpha_{\gamma}$ and $A_{\gamma}$ and introduce the quantity:

$$
A_{\gamma}^{*}=A_{\gamma}\left(\frac{d \tilde{\lambda}}{d \lambda}\right)^{\frac{2}{D-2}}=\frac{\tilde{\lambda}^{2}}{\lambda^{2}}\left(\frac{\Delta}{\Delta_{S}}\right)^{\frac{2}{D-2}}
$$

we can recast Equation (6) as:

$$
[\theta]_{q}=\sqrt{\alpha_{\gamma}}\left[\theta+(D-2) \frac{d}{d \lambda} \ln \sqrt{A_{\gamma}^{*}}\right] .
$$

From this, considering the derivative of $\theta$ according to the qmetric:

$$
\begin{aligned}
{\left[\frac{d \theta}{d \lambda}\right]_{q} } & =\left[l^{a} \nabla_{a} \theta\right]_{q} \\
& =\left[l^{a}\right]_{q} \partial_{a}[\theta]_{q} \\
& =\frac{d \lambda}{d \tilde{\lambda}} l^{a} \partial_{a}[\theta]_{q} \\
& =\frac{d \lambda}{d \tilde{\lambda}} \frac{d}{d \lambda}[\theta]_{q} \\
& =\frac{d}{d \tilde{\lambda}}[\theta]_{q},
\end{aligned}
$$

we find:

$$
\begin{aligned}
{\left[\frac{d \theta}{d \lambda}\right]_{q} } & =\alpha_{\gamma} \frac{d \theta}{d \lambda}+\frac{1}{2 \sqrt{\alpha_{\gamma}}}[\theta]_{q} \frac{d \alpha_{\gamma}}{d \lambda}+(D-2) \alpha_{\gamma} \frac{d^{2}}{d \lambda^{2}} \ln \sqrt{A_{\gamma}^{*}} \\
& =\alpha_{\gamma} \frac{d \theta}{d \lambda}+\frac{1}{2}\left[\theta+(D-2) \frac{d}{d \lambda} \ln \sqrt{A_{\gamma}^{*}}\right] \frac{d \alpha_{\gamma}}{d \lambda}+(D-2) \alpha_{\gamma} \frac{d^{2}}{d \lambda^{2}} \ln \sqrt{A_{\gamma}^{*}}
\end{aligned}
$$


In the third equality above, use has been made of $\left[l^{a}\right]_{q}=d x^{a} / d \tilde{\lambda}=(d \lambda / d \tilde{\lambda}) l^{a}$.

Equation (9) is supposed to be the qmetric rate of change of the expansion for the null congruence $\Gamma$. It exhibits quite a close resemblance to the qmetric rate of change of expansion found in [12] for congruences of unit-tangent spacelike/timelike integral curves emerging from $P$ (Equation (22) in that paper), which, when the congruence is specialized to (spacelike/timelike) geodesics (which is the context to which the qmetric (1) refers to), reads:

$$
\left[\frac{d \theta}{d \lambda}\right]_{q}=\alpha \frac{d \theta}{d \lambda}+\frac{1}{2}\left[\theta+(D-1) \frac{d}{d \lambda} \ln \sqrt{A}\right] \frac{d \alpha}{d \lambda}+(D-1) \alpha \frac{d^{2}}{d \lambda^{2}} \ln \sqrt{A},
$$

where $\alpha$ and $A$ are given in Equations (2) and (3). We see that Equations (9) and (10) are obtained one from the other through the replacements $(D-2), \alpha_{\gamma}, A_{\gamma}^{*} \leftrightarrow(D-1), \alpha, A$.

Making use of the explicit expressions for $\alpha_{\gamma}$ and $A_{\gamma}^{*}$ (Equations (4) and (7)), as well as of the convenient expression:

$$
\theta=\frac{D-2}{\lambda}-\frac{d}{d \lambda} \ln \Delta
$$

relating the expansion and the van Vleck determinant in null congruences ([18]; see also [5]), Expressions (8) and (9) of the expansion and of its rate of change can be given the form:

$$
\begin{gathered}
{[\theta]_{q}=\frac{D-2}{\tilde{\lambda}}-\frac{d}{d \tilde{\lambda}} \ln \Delta_{S},} \\
{\left[\frac{d \theta}{d \lambda}\right]_{q}=-\frac{D-2}{\tilde{\lambda}^{2}}-\frac{d^{2}}{d \tilde{\lambda}^{2}} \ln \Delta_{S} .}
\end{gathered}
$$

In these (exact) expressions, any dependence of $[\theta]_{q}$ and $[d \theta / d \lambda]_{q}$ on $\alpha_{\gamma}$ and $A_{\gamma}^{*}$ has been translated into a dependence on $\tilde{\lambda}$ and $\Delta_{S}$. Comparison with Equation (11), and its derivative:

$$
\frac{d \theta}{d \lambda}=-\frac{D-2}{\lambda^{2}}-\frac{d^{2}}{d \lambda^{2}} \ln \Delta
$$

shows that the effective expansion and its effective rate of change at $p$ with $\lambda=\lambda(p, P)$ turn out to be nothing more than the expansion and its rate of change evaluated at point $\tilde{p}$ on the same null geodesic through $P$ and $p$ with $\lambda(\tilde{p}, P)=\tilde{\lambda}$. From:

$$
\frac{d \theta}{d \lambda}=-\frac{\theta^{2}}{D-2}-\frac{2}{\lambda} \frac{d}{d \lambda} \ln \Delta+\frac{1}{D-2}\left(\frac{d}{d \lambda} \ln \Delta\right)^{2}-\frac{d^{2}}{d \lambda^{2}} \ln \Delta
$$

(upon using (11) in (14)), accordingly we also get:

$$
\left[\frac{d \theta}{d \lambda}\right]_{q}=-\frac{[\theta]_{q}^{2}}{D-2}-\frac{2}{\tilde{\lambda}} \frac{d}{d \tilde{\lambda}} \ln \Delta_{S}+\frac{1}{D-2}\left(\frac{d}{d \tilde{\lambda}} \ln \Delta_{S}\right)^{2}-\frac{d^{2}}{d \tilde{\lambda}^{2}} \ln \Delta_{S}
$$


This fact makes Equations (12) and (13), as well as (16), quite useful when evaluating $[\theta]_{q}$ and $[d \theta / d \lambda]_{q}$ at the coincidence limit. We find:

$$
\begin{aligned}
{[\theta]_{0} } & \equiv \lim _{\lambda \rightarrow 0}[\theta]_{q} \\
& =\frac{D-2}{L}-\frac{d}{d L} \ln \Delta_{L} \\
& =\frac{D-2}{L}-\frac{1}{3} L\left(R_{a b} l^{a} l^{b}\right)_{\mid P}+o\left[L\left(R_{a b} l^{a} l^{b}\right)_{\mid P}\right] \\
& =\frac{D-2}{L}\left[1-\frac{1}{3(D-2)} \delta+o(\delta)\right]
\end{aligned}
$$

and:

$$
\begin{aligned}
{\left[\frac{d \theta}{d \lambda}\right]_{0} } & \equiv \lim _{\lambda \rightarrow 0}\left[\frac{d \theta}{d \lambda}\right]_{q} \\
& =-\frac{D-2}{L^{2}}-\frac{d^{2}}{d L^{2}} \ln \Delta_{L} \\
& =\frac{d}{d L} \lim _{\lambda \rightarrow 0}[\theta]_{q} \\
& =-\frac{D-2}{L^{2}}-\frac{1}{3}\left(R_{a b} l^{a} l^{b}\right)_{\mid P}+o\left[\left(R_{a b} l^{a} l^{b}\right)_{\mid P}\right] \\
& =-\frac{D-2}{L^{2}}\left[1+\frac{1}{3(D-2)} \delta+o(\delta)\right],
\end{aligned}
$$

as well as:

$$
\left[\frac{d \theta}{d \lambda}\right]_{0}=-\frac{[\theta]_{0}^{2}}{D-2}-\frac{2}{L} \frac{d}{d L} \ln \Delta_{L}+\frac{1}{D-2}\left(\frac{d}{d L} \ln \Delta_{L}\right)^{2}-\frac{d^{2}}{d L^{2}} \ln \Delta_{L}
$$

where $\Delta_{L}$ is defined as $\Delta_{L}=\Delta(\bar{p}, P)$ with $\bar{p}$ on $\gamma$ such that $\lambda(\bar{p}, P)=L$, and we used of the expansion ([15,17-19]):

$$
\Delta(p, P)=1+\frac{1}{6} \lambda^{2}\left(R_{a b} l^{a} l^{b}\right)_{\mid P}+o\left[\lambda^{2}\left(R_{a b} l^{a} l^{b}\right)_{\mid P}\right]
$$

of the van Vleck determinant and put $\delta \equiv L^{2}\left(R_{a b} l^{a} l^{b}\right)_{\mid P}$ with the expansions useful when $\delta \ll 1$; this sets a maximum allowed value for $\left(R_{a b} l^{a} l^{b}\right)_{\mid P}$. We see that, whereas classically, i.e., according to $g_{a b}$, both $\theta$ and $d \theta / d \lambda$ diverge when $p \rightarrow P$ (being $\theta \sim \frac{D-2}{\lambda}$ and $\frac{d \theta}{d \lambda} \sim-\frac{D-2}{\lambda^{2}}$ for $\lambda \rightarrow 0$ ), according to the qmetric they both remain finite, the limiting values of $[\theta]_{q}$ and $[d \theta / d \lambda]_{q}$ turning out to be the expressions for $\theta$ and $d \theta / d \lambda$ computed at $\lambda=L$.

This adds, and corresponds, to the non-vanishing of the effective cross-sectional $(D-2)$-dimensional area of $\Gamma$ in the coincidence limit $p \rightarrow P$. Indeed, from:

$$
\begin{aligned}
{\left[d^{D-1} V\right]_{q} } & =\left(\frac{\tilde{\lambda}}{\lambda}\right)^{D-2} \frac{\Delta}{\Delta_{S}} d^{D-2} \mathcal{A} d \lambda \\
& \equiv\left[d^{D-2} \mathcal{A}\right]_{q} d \lambda
\end{aligned}
$$

(Ref. [5], Equation (32), upon using the explicit expression for $A_{\gamma}$ ), where $\left[d^{D-1} V\right]_{q}$ is the effective volume element and $\left[d^{D-2} \mathcal{A}\right]_{q}$ the effective cross-sectional area of the volume element $d^{D-1} V=d^{D-2} \mathcal{A} d \lambda$ of $\Gamma$, we get: 


$$
\begin{aligned}
{\left[d^{D-2} \mathcal{A}\right]_{0} } & \equiv \lim _{\lambda \rightarrow 0}\left[d^{D-2} \mathcal{A}\right]_{q} \\
& =L^{D-2} \frac{1}{\Delta_{L}}(d \chi)^{D-2}
\end{aligned}
$$

where we consider as the cross-sectional area element a $(D-2)$-cube of edge $\lambda d \chi$. This completes what we were searching for.

If we start now from the classical Raychaudhuri equation as applied to our (affinely-parameterized) null congruence $\Gamma$, written as:

$$
\frac{d \theta}{d \lambda}=-\frac{1}{D-2} \theta^{2}-\sigma_{a b} \sigma^{a b}-R_{a b} l^{a} l^{b}
$$

( $\sigma_{a b}$ is shear; the twist is vanishing due to surface-orthogonality) and the use of (15), we get:

$$
\sigma_{a b} \sigma^{a b}+R_{a b} l^{a} l^{b}=\frac{d^{2}}{d \lambda^{2}} \ln \Delta+\frac{2}{\lambda} \frac{d}{d \lambda} \ln \Delta-\frac{1}{D-2}\left(\frac{d}{d \lambda} \ln \Delta\right)^{2},
$$

and, from (16),

$$
\left[\sigma_{a b} \sigma^{a b}\right]_{q}+\left[R_{a b} l^{a} l^{b}\right]_{q}=\frac{d^{2}}{d \tilde{\lambda}^{2}} \ln \Delta_{S}+\frac{2}{\tilde{\lambda}} \frac{d}{d \tilde{\lambda}} \ln \Delta_{S}-\frac{1}{D-2}\left(\frac{d}{d \tilde{\lambda}} \ln \Delta_{S}\right)^{2},
$$

with its coincidence limit:

$$
\lim _{\lambda \rightarrow 0}\left(\left[\sigma_{a b} \sigma^{a b}\right]_{q}+\left[R_{a b} l^{a} l^{b}\right]_{q}\right)=\frac{d^{2}}{d L^{2}} \ln \Delta_{L}+\frac{2}{L} \frac{d}{d L} \ln \Delta_{L}-\frac{1}{D-2}\left(\frac{d}{d L} \ln \Delta_{L}\right)^{2} .
$$

In particular, we can read here the expression for $\left[R_{a b} l^{a} l^{b}\right]_{q}$ and its coincidence limit in the shearless case.

To conclude, we briefly comment on a consequence of the above regarding singularities. Let us consider the spacetime associated with a spherical layer of photons, assumed to be point-like particles, undergoing spherically-symmetric collapse towards a focal point $P$ (we could consider massive particles, as well, but we choose photons to adhere to the results presented above). In our picture, we can look at this as a spherically-symmetric congruence of null geodesics emerging from $P$ and tracked backwards in time, with the further crucial assumption that these geodesics are actual histories of photons, which are then considered as source of spacetime curvature. For these circumstances, the classical description tells us that a singularity unavoidably develops (this is a sort of prototypical case of singularity formation in general relativity). Indeed, photons reach $P$ in a finite variation $\Delta \lambda$ of affine parameter, with diverging energy densities $\rho=E / \mathcal{A}$ (energy per unit transverse area). This means that, in a finite $\Delta \lambda$, photon histories do cease to exist, while some components of the Riemann tensor w.r.t. a basis parallelly-propagated along the geodesics grow without limit, i.e., we have incomplete geodesics corresponding to a parallelly-propagated singularity curvature [6].

According to the qmetric description, in that same $\Delta \lambda$ photon histories keep staying away from $P$ (since the spatial distance from the actual location $p$ of the photon and $P$ according to any canonical observer at $P$ remains no lower than $L$ ), and energy density reaches a maximum insurmountable value $[\rho]_{0}=\lim _{\lambda \rightarrow 0}[\rho]_{q}=E /[\mathcal{A}]_{0}$. Then photon histories do not cease to exist after $\Delta \lambda$, and, using the density $[\rho]_{0}$ as source of Einstein's equations, no components of Riemann in a parallelly-propagated basis are any longer diverging. In this sense, we can say then that the microstructure of spacetime, as captured by the qmetric, removes a classically-blatant curvature singularity.

Assuming $L$ is as small as orders of Planck's length, the density $[\rho]_{0}$ actually challenges the domain of validity of Einstein's equations and the notion of spacetime, as can be envisaged by computing (Equation (21)): 


$$
[\mathcal{A}]_{0}=4 \pi L^{2} \frac{1}{\Delta_{L}}
$$

where $\Delta_{L}=\Delta(\bar{p}, P)$ is finite in spite of being the classical metric singular at $P$ when $\lambda=0(\Delta(\bar{p}, P)$ is indeed computed for the metric configuration associated with $\lambda(\bar{p}, P)=L$, that is, clearly, with $\lambda \neq 0$ ). The qmetric thus embodies that, after $\Delta \lambda$, the photons' spacetime, instead of becoming singular, changes its nature from continuous to discrete and calls for new equations, different from Einstein's, to rule its evolution.

At variance with [11], our derivation does not assume a fixed background spacetime. Indeed, all quantum spacetime effects at $P$ are thought to be subsumed by the qmetric, and the photons that go along the null congruence actively contribute in determining the qmetric at $P$. Due to the complete generality of our model, not much can be said about the specific physical mechanisms that lead to a finite expansion and a finite rate of change of it in the coincidence limit. What we do can say is that this is an effect of quantum geometry, since this is what the qmetric embodies. Our point of view is that in the approach presented here, the specific physical mechanisms in action could be handled only when we have some hint about how to modify Einstein's equations when we are approaching the scale $L$. For a detailed account of the manner in which the formation of a singularity is avoided, one should take into account in an essential manner the influence of the imploding matter itself on the geometry, and this requires the new field equations. What this study seems able to say is simply that at circumstances in which general relativity requires a singularity formation, the granular structure of spacetime as captured by the qmetric requires that no singularity is formed; this on general grounds, whatever the new field equations will be.

Upon comparing these results with those found in LQG and LQC $[9,10]$, in our opinion it is fair to say that, if under isotropic symmetry conditions, the latter are far more definite and accurate in their predictions (e.g., in cosmology, the effective Raychaudhuri equation when followed backwards towards the initial singularity predicts a vanishing of the expansion with a change of sign of the rate of change of the latter, namely a bounce), i.e., the approach presented here seems to have some predictive disadvantages or some loss of accuracy. This however is part of the game. LQG is indeed a specific theory of quantum gravity. Here, instead, we remain as generic as possible when introducing quantum effects on geometry. Another way to look at this is to consider that in LQG the quantization of length is an induced concept. It is a consequence of a quantization procedure based on general relativity (discretization of the classical theory and the search for a quantum theory corresponding to this discretization) [20]. Here, instead, length quantization is meant as a simple primary concept, a basic unavoidable (meaning, it should be present in any quantum theory of gravity) quantum effect, and all the discussion is built on this. When considering singularity issues, the genericity of our approach has its own point of merit. Indeed, the results we obtain, in particular the avoidance of singularity formation, happen to be absolutely general (and a similar comment could be done for [11]). From this, singularity avoidance in quantum gravity turns out to be on an even firmer ground in that it cannot be considered as specific to the quantum theory of gravity one is considering, but happens whichever this theory might be.

Funding: This research received no external funding.

Acknowledgments: I thank S. Chakraborty, D. Kothawala and G. Venturi for having read and made suggestions to earlier versions of the paper.

Conflicts of Interest: The author declares no conflict of interest.

\section{References}

1. Kothawala, D. Minimal length and small scale structure of spacetime. Phys. Rev. D 2013, 88, 104029, arXiv:1307.5618.

2. Kothawala, D.; Padmanabhan, T. Grin of the Cheshire cat: Entropy density of spacetime as a relic from quantum gravity. Phys. Rev. D 2014, 90, 124060, arXiv:1405.4967. 
3. Jaffino Stargen, D.; Kothawala, D. Small scale structure of spacetime: Van Vleck determinant and equi-geodesic surfaces. Phys. Rev. D 2015, 92, 024046, arXiv:1503.03793.

4. Kothawala, D.; Padmanabhan, T. Entropy density of spacetime from the zero point length. Phys. Lett. B 2015, 748, 67-69, arXiv:1408.3963.

5. Pesci, A. Looking at spacetime atoms from within the Lorentz sector. arXiv 2018, arXiv:1803.05726.

6. Hawking, S.W.; Ellis, G.F.R. The Large Scale Structure of Space-Time; Cambridge University Press: Cambridge, UK, 1973.

7. Misner, C.W.; Thorne, K.S.; Wheeler, J.A. Gravitation; W.H. Freeman and Company: New York, NY, USA, 1973.

8. Thorne, K.S. Black Holes E Time Warps; W.W. Norton \& Co.: New York, NY, USA, 1994.

9. Ashtekar, A.; Bojowald, M. Quantum geometry and the Schwarzschild singularity. Class. Quantum Gravity 2006, 23, 391, arXiv:gr-qc/0509075.

10. Bojowald, M. Loop quantum cosmology. Living Rev. Relat. 2005, 8, 11, arXiv:gr-qc/0601085.

11. Das, S. Quantum Raychaudhuri equation. Phys. Rev. D 2014, 89, 084068, arXiv:1311.6539.

12. Kothawala, D. Intrinsic and extrinsic curvatures in Finsleresque spaces. Gen. Relat. Gravit. 2014, 46, 1836, arXiv:1406.2672.

13. Van Vleck, J.H. The correspondence principle in the statistical interpretation of quantum mechanics. Proc. Natl. Acad. Sci. USA 1928, 14, 178-188. [CrossRef] [PubMed]

14. Morette, C. On the definition and approximation of Feynman's path integrals Phys. Rev. 1951, 81, 848.

15. DeWitt, B.S.; Brehme, R.W. Radiation damping in a gravitational field. Ann. Phys. 1960, 9, 220-259. [CrossRef]

16. DeWitt, B.S. The Dynamical Theory of Groups and Fields; Gordon and Breach: New York, NY, USA, 1965.

17. Christensen, S.M. Vacuum expectation value of the stress tensor in an arbitrary curved background: The covariant point-separation method. Phys. Rev. D 1976, 14, 2490-2501. [CrossRef]

18. Visser, M. Van Vleck determinants: Geodesic focussing and defocussing in Lorentzian spacetimes. Phys. Rev. D 1993, 47, 2395-2402. [CrossRef]

19. Poisson, E.; Pound, A.; Vega, I. The motion of point particles in curved spacetime. Living Rev. Relat. 2011, 14, 7, arXiv:1102.0529.

20. Rovelli, C.; Vidotto, F. Covariant Loop Quantum Gravity; Cambridge University Press: Cambridge, UK, 2015.

(C) 2018 by the authors. Licensee MDPI, Basel, Switzerland. This article is an open access article distributed under the terms and conditions of the Creative Commons Attribution (CC BY) license (http:/ / creativecommons.org/licenses/by/4.0/). 